\title{
Invasion and persistence of Salmonella in human fibroblasts positive or negative for endogenous HLA B27
}

Hans-Iko Huppertz, Jürgen Heesemann

\begin{abstract}
Objective-Analysis of the interaction of enteropathogenic bacteria with HLA B27 transfected murine fibroblasts showed a specific influence of HLA B27 on microbial invasiveness. This possible novel mechanism for the action of HLA B27 should be verified by using endogenous HLA B27 positive and negative human fibroblasts as a model for the direct interaction of arthritogenic bacteria and host cells.

Methods-Fibroblasts were obtained from healthy donors positive or negative for HLA B27; cultivated as monolayers; and infected with Salmonella enterica serovar enteritidis.

Results-Invasion and survival of bacteria in human cells was not influenced by the presence of HLA B27. Enhancement of HLA class I molecule expression by treatment of cultures with interferon gamma decreased invasion and survival of bacteria in both HLA B27 positive and negative cells. After disappearance of live bacteria lipopolysaccharide antigens persisted within cells.

Conclusion-Endogenous HLA B27 does not modulate the direct interaction of Salmonella with human cells. Nonprofessional phagocytes are able to limit bacterial survival in cells, and interferon gamma accelerates killing of bacteria, but arthritogenic antigens persist after disappearance of live bacteria.
\end{abstract}

(Ann Rheum Dis 1997;56:671-676)

Reactive arthritis triggered by infection with enteropathogenic bacteria is a frequent cause of acute inflammatory joint disease. ${ }^{1}$ One to three weeks after infection with or without gastroenteritis patients may suffer from mild arthralgias to severe polyarthritis. ${ }^{2}$ After a few weeks to months most patients recover, but some may have ongoing arthritis or even progress to ankylosing spondylitis. ${ }^{3}$

Spondyloarthropathies including reactive arthritis are associated with the presence of the human MHC class I antigen HLA B27. ${ }^{4}$ However, it is unknown how HLA B27 and the different bacteria might cooperate to initiate arthritis. $^{5}$ Although constitutive HLA B27 positive and negative human monocytes did not differ in their ability to process and express Yersinia antigen, ${ }^{6}$ the monocytic cell line U937 demonstrated impaired elimination of Salmonella after three to five days ${ }^{7}$ and the B cell line $\mathrm{C} 1 \mathrm{R}$ showed decreased invasion ${ }^{8}$ when these cell lines had been transfected with human HLA B27.

Mouse fibroblast $\mathrm{L}$ cells transfected with human HLA B27 were shown to have a decreased invasiveness for Salmonella ${ }^{9}{ }^{10}$ and a prolonged trypsin elution after infection with Yersinia. ${ }^{11}$ However, another research group found no change in invasion caused by the transfection of HLA B27, but an impaired killing of internalised bacteria ${ }^{12}$ possibly because of impaired nitric oxide production. ${ }^{13}$ We could confirm a decreased invasiveness of Yersinia in HLA B27 transfected L cells, but found no influence of endogenous HLA B27 on invasion or persistence of Yersinia in primary human cell lines expressing constitutive HLA B27. ${ }^{14}$ This study was focused upon the following issues: (a) comparison of invasion and persistence of Salmonella in HLA B27 positive and negative human fibroblasts; (b) fate of live and dead bacteria in these cells; (c) effect of interferon gamma on the Salmonella infection in human fibroblasts.

\section{Methods}

CELLS

Primary human fibroblasts derived from skin and maternal abortion material were kindly donated by Dr Höhn (Institute of Human Genetics, University of Würzburg, Würzburg, Germany) and grown in RPMI 1640 supplemented with $10 \%$ fetal calf serum and glutamine and propagated with trypsin, as has been described. ${ }^{15}$ Cells were used for the experiments during passages 4 to 11 . Murine fibroblast L cells, transfected with human HLA B27 or not transfected, were donated by Dr E Herrmann, Mainz, Germany, with the kind permission of Dr J Taurog, Dallas, Texas. ${ }^{9}$

HLA B27

Cells were screened for the presence of HLA B27 by polymerase chain reaction kit using specific primers ${ }^{16}$ and following the manufacturer's instructions (Dynal, Oslo, Norway). Expression of HLA B27 was found by indirect immunofluorescence and flow cytometry using a FACScan flow cytometer (BectonDickinson, California) and two different antibodies to HLA B27 (HLA-ABC-m3; Chemicon International, Temecula, California; Dianova, Hamburg, Germany). We established 20 HLA B27 negative and six HLA B27 positive cell lines 

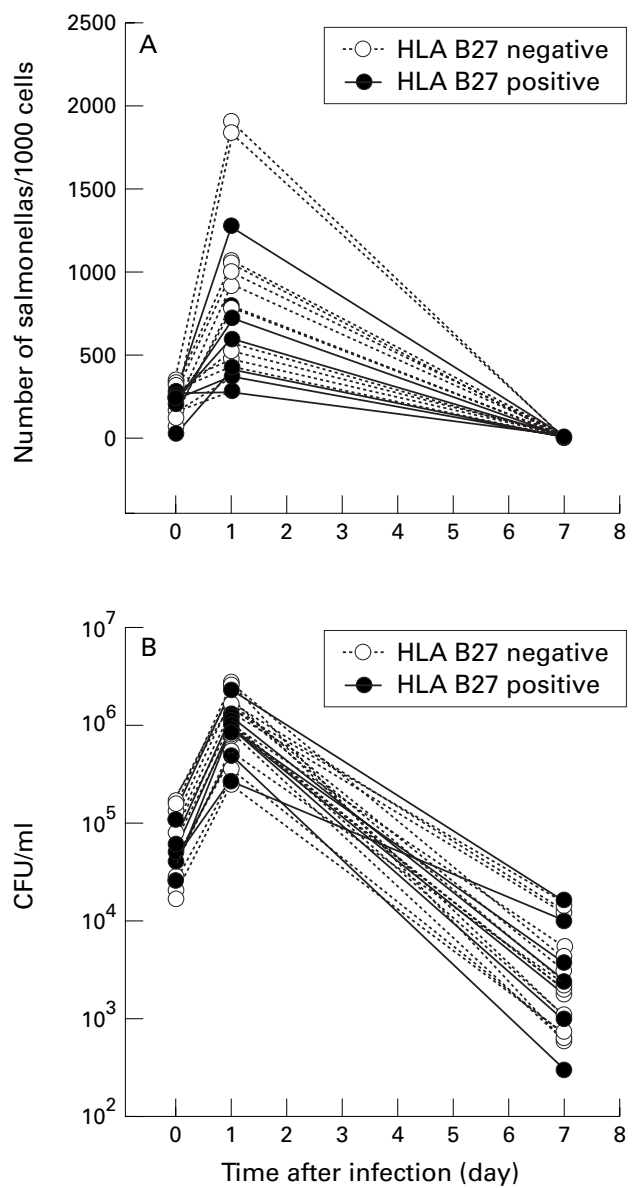

Figure 1 Influence of HLA B27 on invasion and survival of Salmonella enterica serotype enteritidis in 26 primary human fibroblast lines, six of which were $H L A$ B27 positive and 20 HLA B27 negative. (A) Number of salmonellas per 100 cells after the infection (invasion) and one and seven days later (survival). Median (range) in HLA B27 positive/ negative cells were: day 0:210 (30-268) / 251 (68-348); day 1: 505 (282-1280) / 794 (282-1910); day 7: 1 (1-6) / 2 (1-4). (B) Colony forming units per monolayer (CFU/ml) after the infection and one and seven days later. Median (range) in HLA B27 positive / negative cells were: day 0: $4.6 \times 10^{4}(2.6-11$ $\left.\times 10^{4}\right) / 7.4 \times 10^{4}\left(1.7-17 \times 10^{4}\right)$; day $1: 9.5 \times 10^{5}$ $\left(2.7-23 \times 10^{5}\right) / 12.0 \times 10^{5}\left(2.5-27 \times 10^{5}\right)$; day $7: 30 \times$ $10^{2}\left(3.1-160 \times 10^{2}\right) / 22 \times 10^{2}\left(6.0-150 \times 10^{2}\right)$. Comparing the groups of HLA B27 positive and negative cell lines for the number of salmonellas per 100 cells and the CFU/ml at the three time points by $U$ test did not yield any significant differences.

from 26 people. Three of six HLA B27 positive cells have been described before. ${ }^{14}$

BACTERIA

Patient isolates of Salmonella enterica serotype enteritidis and, for comparison, Yersinia enterocolitica 0.3 were used. These are the dominant serotypes found in patients with reactive arthritis in Europe. Salmonella was grown in brain heart infusion broth (BHIB) overnight. After diluting the culture 1:20 cultivation was continued for three hours at $37^{\circ} \mathrm{C}$ (mid-log phase). Yersinia was grown in BHIB, as has been described. ${ }^{17}$ The number of surviving bacteria was determined as colony forming units per monolayer by lysis of cells of infected monolayers with $0.5 \%$ tergitol and subsequent plating on Müller-Hinton agar. Giemsa staining of monolayers showed intracellular bacteria
Table 1 Conversion of intact bacterial rods into bacterial 'ghosts', seemingly intact bacterial rods but devoid of DNA. Determination of the percentage of primary human fibroblasts containing bacterial antigen and the percentage of the found bacterial rods that are negative for DNA by propidium iodide. Comparison of cell lines positive or negative for $H L A$ B 27

\begin{tabular}{lllll}
$\begin{array}{l}\text { Days } \\
\text { after } \\
\text { infection }\end{array}$ & $\begin{array}{l}\text { Percentage of cells positive } \\
\text { by immunofluorescence for } \\
\text { Salmonella antigen }\end{array}$ & $\begin{array}{l}\text { Percentage of bacterial } \\
\text { 'ghosts' of all rods }\end{array}$ \\
\hline & HLA B27 & HLA B27 & HLA & HLA B27 \\
& neg & pos & B27neg & pos \\
day 0 & 50 & 60 & 0 & 0 \\
day 4 & 70 & 60 & 80 & 70 \\
day 7 & 75 & 85 & 90 & 90 \\
day 14 & 85 & 80 & 100 & 100 \\
day 21 & 90 & 90 & 100 & 100 \\
\hline
\end{tabular}

expressed as the number of bacteria per 100 cells. Salmonella antigen was demonstrated by indirect immunofluorescence using a rabbit polyclonal serum against Salmonella group D (Behring, Marburg, Germany) or a polyclonal monospecific rabbit serum against Salmonella enterica prepared in our laboratory and used at a concentration of $1: 10000^{18}$ and a FITC tagged goat antirabbit immunoglobulin preparation diluted 1:50 (Dianova, Hamburg, Germany). Both serum samples gave similar results. For control uninfected cells were stained or the primary antibody was replaced by normal rabbit serum (Sigma). Propidium iodide (Sigma) was used for staining DNA. Recombinant human interferon gamma was a kind gift from Rentschler (Laupheim, Germany).

INFECTION PROTOCOL

Before infection salmonellas were adjusted to a multiplicity of infection (MOI) of 50 salmonellas per cell in RPMI 1640 cell culture medium. This infectious dose was used as it was not toxic for cells and allowed for a sufficient number of bacteria to enter the cells. MOIs of 0.01 up to 1000 did not increase the number of detached cells in the supernatant nor decrease the number of cells in the culture dishes immediately after the infection and two days later. Subconfluent cells were infected at $37^{\circ} \mathrm{C}$ with the bacterial suspension for 30 minutes, then washed and incubated for two hours in medium containing $16.2 \mu \mathrm{g} / \mathrm{ml}$ gentamicin

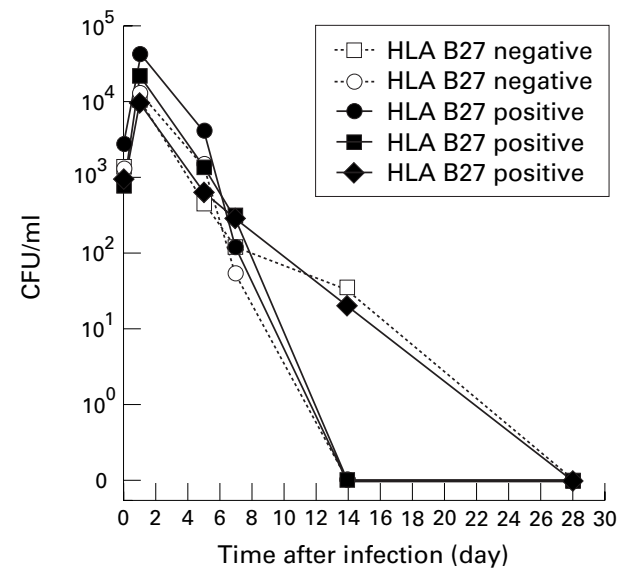

Figure 2 Persistent infection of Salmonella enterica serotype enteritidis in primary human fibroblasts. Three cell lines are HLA B27 positive, two lines HLA B27 negative. 

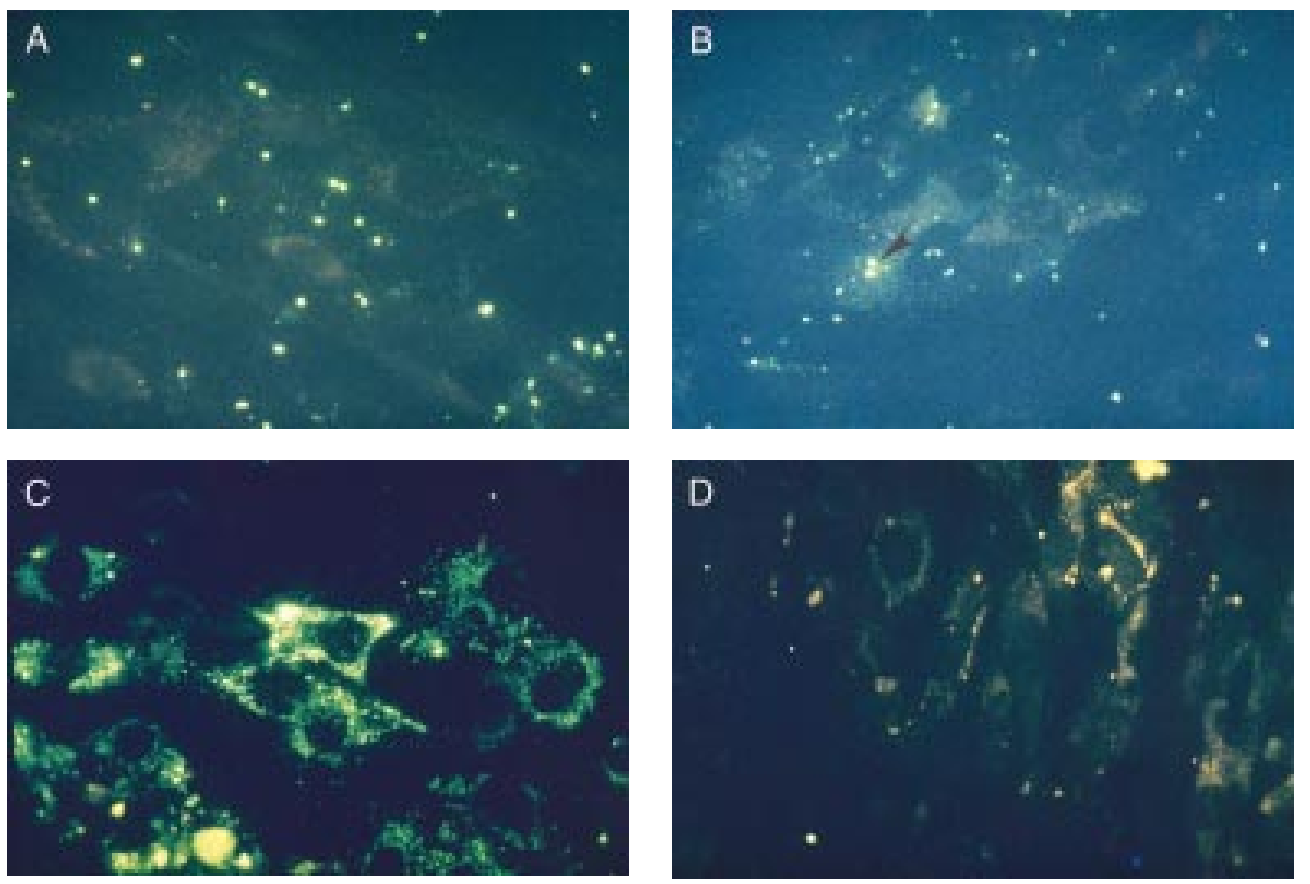

Figure 3 Salmonella antigen in primary human fibroblasts by indirect immunofluorescence staining for Salmonella lipopolysaccharide antigens directly after infection and two hours of antibiotic treatment $(A)$ and seven (B) and 21 days later (C). Initially intact bacterial rods prevail. After seven days aggregates of cytoplasmic antigen deposits can be seen (arrowhead). After 21 days, at a time when the culture had become sterile, bacterial rods can no longer be identified, but finely dispersed antigen deposit can be seen in the cells. For control a parallel culture was stained with normal rabbit serum after 21 days of infection (D); to show cell morphology exposure time was doubled in comparison with fig 3C. Staining with the normal rabbit serum shows no green colour, but only faint yellow non-specific background staining.

(Merck, Darmstadt, Germany) to kill extracellular bacteria. After a further washing step, cells were grown in medium with $6.5 \mu \mathrm{g} / \mathrm{ml}$ gentamicin. The minimal inhibitory concentration for gentamicin was $1.25 \mu \mathrm{g} / \mathrm{ml}$. At gentamicin concentrations of $16.2 \mu \mathrm{g} / \mathrm{ml}$ or greater, the number of salmonellas that could be grown from lysed monolayers decreased after five days of culture. Pretreatment of cells for five days with gentamicin at $16.2 \mu \mathrm{g} / \mathrm{ml}$ or greater followed by the standard protocol diminished intracellular survival of salmonellas; pretreatment with $6.5 \mu \mathrm{g} / \mathrm{ml}$ or $3.3 \mu \mathrm{g} / \mathrm{ml}$ or no treatment had no effect. Therefore, the standard protocol permitted protection of internalised salmonellas from gentamicin treatment and killing of extracellular salmonellas.

\section{STATISTICS}

Statistical analysis was done with the MannWhitney U test or the two way analysis of variance with repeated measures (ANOVA).

\section{Results}

When mouse L cells with or without transfected HLA B27 were infected with salmonellas the number of bacteria detected in three different experiments by Giemsa stain or by colony forming units was inferior in transfected cells after incubation of cells with bacteria (invasion) and one and seven days later (survival). To investigate if this was a specific effect of HLA B27 or due to the artificial system of non-human cells transfected with the human gene, we infected 26 primary human fibroblast lines, six of which were HLA B27 positive, with Salmonella enterica.
The number of salmonellas per 100 cells and the colony forming units after incubation and one and seven days later were not different between HLA B27 positive and negative cells (fig 1).

To assess if the effect of HLA B27 on the fate of Salmonella in human cells might become apparent at a later stage of infection, cultures
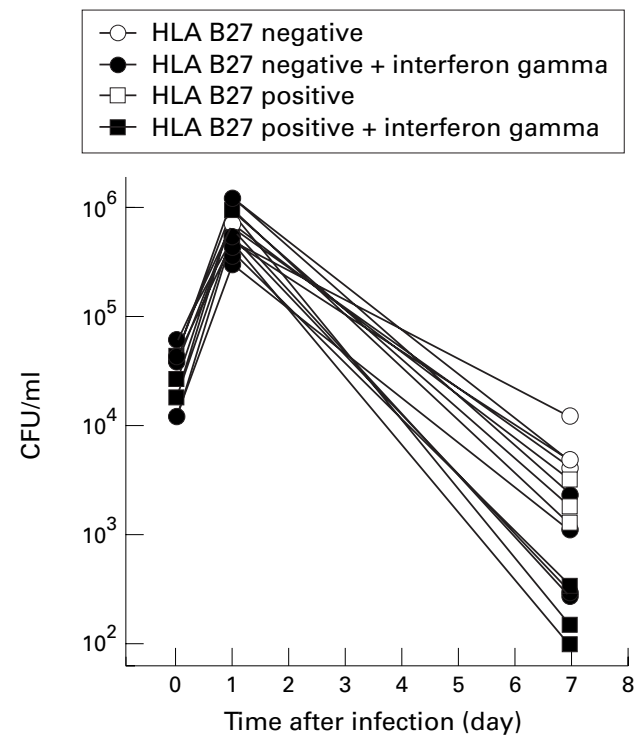

Figure 4 Effect of treatment with interferon gamma on the survival of Salmonella in primary human fibroblasts.

Seven cell lines were infected with Salmonella enterica and then treated with $1000 \mathrm{IU} / \mathrm{ml}$ of interferon gamma or left untreated. The three HLA B27 positive cell lines are displayed with square symbols, the four HLA B27 negative cell lines with round symbols. Using ANOVA to compare treated and untreated cells treatment effect $(p<0.001)$ and interaction $(p<0.0001)$ were significantly different. 
- HLA B27 negative

- HLA B27 negative + interferon gamma $-\square-$ HLA B27 positive

- HLA B27 positive + interferon gamma

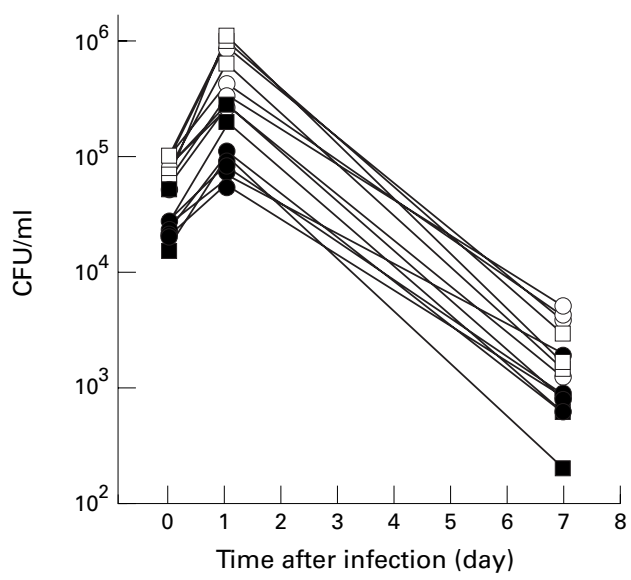

Figure 5 Effect of pre-treatment with interferon gamma on the invasion of Salmonella in primary human

fibroblasts. Seven cell lines were treated with $1000 \mathrm{IU} / \mathrm{ml}$ of interferon gamma for 48 hours or left untreated and then infected with Salmonella enterica. The three HLA B27 positive cell lines are displayed with square symbols, the four HLA B27 negative cell lines with round symbols. Using ANOVA to compare treated and untreated cells treatment effect was highly significant $(p<0.000005)$, but interaction was not, confirming the significant influence of interferon gamma on invasion.

were incubated for up to one month after infection. Bacterial titres increased during the first day after invasion, but had decreased at day 5 after infection. Culturable salmonellas could no longer be isolated after two to four weeks (fig 2). All cultures, however, carried intracellular Salmonella antigen detected by immunofluorescence at a time when live bacteria had already vanished (fig 3 ). These results did not differ between HLA B27 positive and negative cells. The results were reproducible in two subsequent experiments. To investigate the transition from cultivable bacterium to antigenic debris cultures were double stained for the presence of DNA and Salmonella antigen. After 24 hours a median of $35 \%$ (range $10-60 \%$ ) of salmonellas were converted to bacterial 'ghosts', seemingly intact bacterial rods, but devoid of DNA. Table 1 shows the time course of 'ghost' development. These results did not differ between HLA B27 positive and negative cells.

To assess if increased expression of HLA B27 might influence intracellular survival of Salmonella, cells were treated with $1000 \mathrm{IU} / \mathrm{ml}$ of interferon gamma after infection. After seven days of infection bacterial titres were significantly lower in interferon gamma treated cultures than in untreated control cultures (fig 4). However, there was no difference between HLA B27 positive and negative cells. The percentage of cells containing Salmonella antigen was not changed by treatment with interferon gamma. A repeat experiment yielded similar results. To increase the expression of HLA B27 already at the time of infection cells were treated with interferon gamma for 48 hours before infection with Salmonella: invasion of bacteria was significantly decreased to about a third of the parallel untreated cultures, but there was no difference between HLA B27 positive and negative cells (fig 5). A repeat experiment yielded similar results. The percentage of cells containing Salmonella antigen after invasion was reduced by pretreatment with inteferon gamma from $60-80 \%$ to 20 $40 \%$. This difference was still apparent seven days after the infection.

To compare Salmonella and Yersinia infections, cells were infected in parallel with these pathogens. Initially Salmonella titres were significantly higher, but decreased more rapidly than Yersinia titres. A repeat experiment yielded similar results.

\section{Discussion}

The results from the laboratories of $\operatorname{Inman}^{8-11}$ and Granfors, ${ }^{72} 13$ showing a modulation of interaction of enteropathogenic bacteria with murine cells that express transfected human HLA B27, prompted us to try to verify their conclusions by using endogenous HLA B27 positive human cells. In this report we have shown that, in contrast with transfected murine cells, endogenous HLA B27 positive primary human fibroblasts do not differ from HLA B27 negative cells in their ability to support invasion and persistence of Salmonella.

Our results are in accordance with a recent presentation from Vancouver showing no difference between HLA B27 positive and negative cells in their ability to support invasion by Salmonella or Yersinia ${ }^{19}$ and with results from our laboratory of Yersinia infection in primary human fibroblasts. ${ }^{14}$ These results cast doubt on the hypothesis that HLA B27 might modulate the interaction of enteropathogenic bacteria and host cells. ${ }^{912}$ Although transfected cells allow for the convenient control of experimental conditions, the random insertion of a xenogenetic molecule separated from its normal chromosomal environment and detached from its expression control may lead to artificial conditions unrelated to those found in patients' cells. On the other hand, the experimental design chosen for this study is artificial as it used fibroblast lines that may have changed their phenotype during culture. We believe that primary human cell lines are closer to the situation found in patients than transfected murine cells.

In a previous study we have shown that human cells expressing endogenous HLA B27 have a lower expression of HLA B27 than transfected mouse cells. ${ }^{14}$ The overexpression of HLA B27 in transfected murine cells might result in changed mechanisms of infection. Overexpression of HLA B27, however, is probably not the mechanism whereby the transfected HLA B27 genes modulate the infection in murine cells as we have shown in a previous study that treatment with interferon gamma is able to increase and equalise HLA B27 expression in transfected and endogenous cells without changing invasion of Yersinia. ${ }^{14}$ However, this overexpression is an indicator of a changed HLA B27 metabolism in these transfected murine cells. Results from Vancouver showing no influence of transfected HLA 
B27 on bacterial invasion in human cells ${ }^{19}$ indicate that the disturbing factor is not the transfection of human HLA B27 itself, but the murine recipient cell that changes invasion of bacteria. In line with this assumption are results from human HLA B27 transgenic rats in which arthritis development depends on the HLA B27 gene copy number, and not expression of HLA B27. Therefore, although transfected cells have been shown to be a valuable experimental tool, we question the relevance of data obtained from transfected murine cells for the infectious pathogenesis of HLA B27 associated diseases.

Interestingly, although fibroblasts are nonprofessional phagocytes they were able to eliminate intracellular bacteria. Salmonella antigens, however, could still be detected at the time when all bacteria had been killed. This antigen load was not diminished when killing of bacteria was accelerated by treatment with interferon gamma. Only when interferon gamma was added before infection could the percentage of cells carrying lipopolysaccharide antigens be reduced. In patients with reactive arthritis interferon gamma found in synovial tissue ${ }^{20}$ probably is secreted after infection and thus may not be able to diminish the antigen load nor shorten the duration of arthritis. In contrast with fibroblasts, macrophages show apoptosis within a few hours after infection with Salmonella. ${ }^{21}$ Salmonella enters the cell into an alternative phagosome, which is unable to kill the bacteria and then forms large vacuoles by fusion of several of these phagosomes. ${ }^{21}$ Bactericidal mechanisms of fibroblasts have not yet been elucidated. To determine this important aspect of pathogenesis ultrastructural investigations are under way in our laboratory.

The comparison of infections with Yersinia and Salmonella showed longer survival of Yersinia in human fibroblasts. In contrast with Yersinia, Salmonella has been shown to induce the formation of filamentous structures in epithelial cells. ${ }^{22}$ It could be argued that these filamentous structures might render intracellular salmonellas more vulnerable to gentamicin treatment. However, as these structures were not accessible to incoming material from the external medium ${ }^{22}$ it is unlikely that gentamicin might have gained access to intracellular bacteria. In addition, pretrials to this study had shown that the concentrations of gentamicin used in these experiments did not affect the number of culturable salmonellas. Finally, other authors had used much higher concentrations of gentamicin than we did. ${ }^{21}{ }^{23} \mathrm{Al}-$ though similar in many respects the interactions of Salmonella and Yersinia with fibroblasts are probably very different at the molecular level ${ }^{22}$ and the longer survival of Yersinia in fibroblasts and the diminished invasion of Salmonella after pretreatment with interferon gamma, seen in this study, might be related to fundamental differences in invasion and intracellular survival. ${ }^{24}{ }^{25}$ The similarity of the results on the influence of HLA B27 on invasion and survival of Yersinia and Salmonella despite fundamental differences at the molecular leve ${ }^{25}$ supports the notion that HLA B27 is not important in this interaction.
The results presented in this paper and previous results ${ }^{14}$ offer a comprehensive hypothesis for the pathogenesis of reactive arthritis caused by enteropathogenic bacteria infection. Soon after intestinal infection bacteria might spread through the body of the host and persist for a few days to weeks in resident fibroblastlike cells of synovial tissue. The host defence, including the production of interferon gamma ${ }^{26}$ and other cytokines, ${ }^{20}$ results in killing of the bacteria. However, the undegradable bacterial antigens ${ }^{27}$ remain within the joint and might be liberated from infected cells to induce an inflammatory response. Thus, a few weeks after infection, the host defence might initiate arthritis. At this time traces of degraded bacterial $\mathrm{DNA}^{28}$ and bacterial antigens can be found in synovial fluid ${ }^{29}$ and synovial tissue of patients with reactive arthritis, ${ }^{30}$ but culturable bacteria are not present. However, the role of HLA B27 in this scenario remains unclear.

We are grateful to Martina Zinser for excellent technical assistance and to Dr I Haubitz, Rechenzentrum, University of Würzburg, for help with statistics. This work was supported by Deutsche Forschungsgemeinschaft (Hu 405) and the Bundesministerium für Bildung und Forschung (ZKF-A2).

1 Locht H, Kihlstrom E, Lindstrom FD. Reactive arthritis after Salmonella among medical doctors, study of an outbreak. J Rheumatol 1993;20:845-8.

2 Mattila L, Lerisalo-Repo M, Koskimies S, Granfors K, Siitonen A. Reactive arthritis following an outbreak of Salmonella infection in Finland. Br J Rheumatol 1994;33:113641 .

3 Thomson GT, DeRubeis DA, Hodge MA, Rajanayagam C, Inman RD. Post-Salmonella reactive arthritis: late clinical sequelae in a point source cohort. Am J Med 1995;98:1321

4 Thomson GT, Minenko A, Schroeder ML. Host risk factors for the development of reactive arthritis: a family study. J Rheumatol 1993;20:1350-2.

5 Hughes RA, Keat AC. Reiter's syndrome and reactive arthritis: a current view. Semin Arthritis Rheum 1994;24: 190-210.

6 Wuorela M, Jalkanen S, Toivanen P, Granfors K. Yersinia lipopolysaccharide is modified by human monocytes. Infect Immun 1993;61:5261-70.

7 Laitio P, Virtala M, Salmi M, Pelliniemi LJ, Yu DTY, Granfors $\mathrm{K}$. Impaired elimination of salmonella within HLA B27-transfected human monocytic U U

8 Inman RD, Chiu B, Pavne U. HLA B27 modulates interaction of Salmonella typhimurium with class I HLAtransfected C1R cells. [Abstract]. Arthritis Rheum 1996; 39:S297.

9 Kapasi K, Inman RD. HLA B27 expression modulates gram-negative bacterial invasion into transfected L cells. J Immunol 1992;148:3554-9.

10 Kapasi K, Inman RD. ME1 epitope of HLA B27 confers class I-mediated modulation of gram-negative bacterial invasion. J Immunol 1994;153:833-40.

11 Inman RD, Payne U, Chiu B. B27 and bacteria: studies on adherence and bacterial interactions with trans

12 Virtala M, Kirveskari J, Gransfors K. HLA B27 modulates intracellular life of salmonella in transfected $\mathrm{L}$ cells. intracellular life of salmonella in transfect

13 Virtala M, Kirveskari J, Gransfors K. Nitric oxide production in HLA B27-transfected mouse fibroblasts infected with Salmonella enteritidis. [Abstract]. Arthritis Rheum 1996;39:S121.

14 Huppertz HI, Heesemann J. The influence of HLA B27 and interferon-gamma on the invasion and persistence of yersinia in primary human fibroblasts. Med Microbiol Immunol 1996;185:163-70.

15 Huppertz HI, Chantler JK. Restricted mumps virus infection of cells derived from normal human joint tissue. J Gen Virol 1991;72:339-47.

16 Olerup O. HLA B27 typing by a group-specific PCR amplification. Tissue Antigens 1994;43:253-6.

17 Huppertz HI, Heesemann J. Experimental Yersinia infection of human synovial cells: persistence of live bacteria and of human synovial cells: persistence of live bacteria and generation of bacterial antigen deposits including "ghosts",
nucleic acid-free bacterial rods. Infect Immun 1996;64: 1484-7. 
18 Huppertz HI, Karch H, Heesemann J. Diagnostic value of synovial fluid analysis in children with reactive arthritis. Rheumatol Int 1995;15:167-70.

19 Ortiz Alvarez O, Finlay BB, Yu D, Petty RE. HLA B27 does not influence invasion of arthritogenic bacteria into human cells. [Abstract]. Arthritis Rheum 1996;39:S162.

20 Simon AK, Seipelt E, Sieper J. Divergent T cell cytokine patterns in inflammatory arthritis. Proc Natl Acad Sci USA patterns in inflam

21 Lindgren SW, Stojilkovic I, Heffron F. Macrophage killing is an essential virulence mechanism of Salmonella typhimurium. Proc Natl Acad Sci USA 1996;93:4197-201.

22 Garcia del Portillo F, Zwick MB, Leung KY, Finlay BB. Salmonella induces the formation of filamentous structures containing lysosomal membrane glycoproteins in epithelial cells. Proc Natl Acad Sci USA 1993;90:10544-8.

23 Guilloteau LA, Wallis TS, Gautier AV, MacIntyre S, Platt DJ, Lax AJ. The Salmonella virulence plasmid enhances Salmonella-induced lysis of macrophages and influences inflammatory responses. Infect Immun 1996;64:3385-93.

24 Garcia del Portillo F, Finlay BB. The varied lifestyles of intracellular pathogens within eukaryotic vacuolar compartments. Trends Microbiol 1995;3:373-80.
25 Galan JE. Interactions of bacteria with non-phagocytic cells. Curr Opinion Immunol 1994;6:590-5.

26 Schlaak J, Hermann E, Ringhofer M, Probst P, Gallati H, Meyer zum Büschenfelde KH, et al. Predominance of Th1type $\mathrm{T}$ cells in synovial fluid of patients with yersiniatype $T$ cells in synovial fluid of patients with yersinia-
induced reactive arthritis. Eur J Immunol 1992;22:2771-6.

27 Henderson B, Poole S, Wilson M. Bacterial modulins: a novel class of virulence factors which cause host tissue pathology by inducing cytokine synthesis. Microbiol Rev 1996;60:316-41.

28 Nikkari S, Möttönen T, Saario R, Yli-Kerttula U, LeirisaloRepo M, Laitio P, et al. Demonstration of Salmonella DNA in the synovial fluid in reactive arthritis. [Abstract]. Arthritis Rheum 1996;39:S185.

29 Granfors K, Jalkanen S, Lindberg AA, Mäki-Ikola Outi, von Essen R, Lahesmaa-Rantala R, et al. Salmonella lipopolysaccharide in synovial cells from patients with reactive arthritis. Lancet 1990;335:685-8.

30 Merilahti-Palo R, Söderström KO, Lahesmaa-Rantala R, Granfors K, Toivanen A. Bacterial antigens in synovial biopsy specimens in yersinia triggered reactive arthritis. Ann Rheum Dis 1991;50:87-90. 